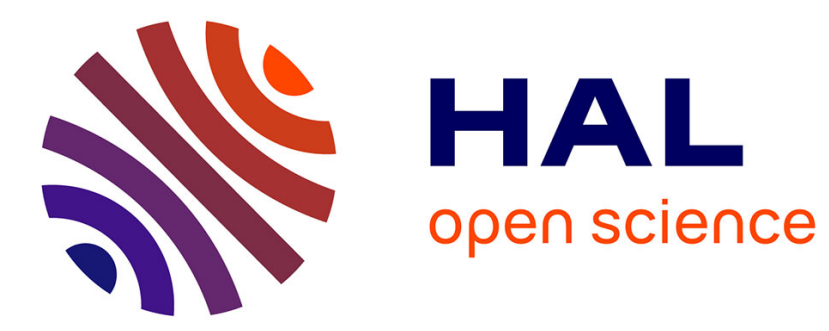

\title{
Tutorial on arbitrary and state-dependent sampling
}

Christophe Fiter, Hassan Omran, Laurentiu Hetel, Jean-Pierre Richard

\section{To cite this version:}

Christophe Fiter, Hassan Omran, Laurentiu Hetel, Jean-Pierre Richard. Tutorial on arbitrary and state-dependent sampling. 13th European Control Conference, Jun 2014, Strasbourg, France. pp.1440-1445, 10.1109/ECC.2014.6862643 . hal-01194572

\section{HAL Id: hal-01194572 \\ https://inria.hal.science/hal-01194572}

Submitted on 7 Sep 2015

HAL is a multi-disciplinary open access archive for the deposit and dissemination of scientific research documents, whether they are published or not. The documents may come from teaching and research institutions in France or abroad, or from public or private research centers.
L'archive ouverte pluridisciplinaire HAL, est destinée au dépôt et à la diffusion de documents scientifiques de niveau recherche, publiés ou non, émanant des établissements d'enseignement et de recherche français ou étrangers, des laboratoires publics ou privés. 


\title{
Tutorial on arbitrary and state-dependent sampling
}

\author{
Christophe Fiter $^{\dagger}$, Hassan Omran ${ }^{\dagger}$, Laurentiu Hetel ${ }^{\dagger}$, and Jean-Pierre Richard ${ }^{\dagger, \ddagger}$,
}

\begin{abstract}
This tutorial, presents basic concepts and recent research directions about sampled-data systems. We focus mainly on the stability of systems with time-varying sampling intervals. Without being exhaustive, which would be neither possible nor useful, we try to give a structural survey of what we think to be the main results and issues in this domain.

Index Terms-networked control systems, time-varying sampling, dynamic sampling, self-triggering, event-triggering.
\end{abstract}

\section{INTRODUCTION}

In the literature, the analysis and design of sampled-data systems with periodic sampling is a well established domain. In the monographs [16], [26], [71], advanced topics such as optimal control, robust controller design, identification, etc. can be found. In practice however, it is difficult to maintain a constant sampling rate during the real-time control of physical systems. Embedded and networked systems are often required to share a limited amount of computational and transmission resources between different applications. This may lead to fluctuations of the sampling interval, due to the interaction between real-time control algorithms and task / communication scheduling protocols [15], [36], [73], [84]. From the control theory point of view, these variations in the sampling interval need to be treated in a robust manner since they may have a destabilizing effect if they are not properly taken into account [83], [46]. Furthermore, aperiodic controller implementations may actually have interesting properties in distributed control applications, when explicitly evaluating energy, computation and communication costs [11], [14], [14], [33]. The new trend in control is to intentionally modify the sampling interval as an additional control parameter using event- and self-triggering control schemes.

This tutorial will present basic concepts and research directions for systems with time-varying sampling intervals. After a short presentation of sampled-data systems (Section II), qualitative properties of sampled-data system are indicated in Section III. The main results concerning the analysis of systems with time-varying sampling intervals will be presented in Section IV. Finally, emerging research directions concerning the reduction of sampling events will be introduced in Section V.

The research leading to these results has received funding from the European Community's 7th Framework Programme (grant agreement No 257462) HYCON2 Network of Excellence and the Interreg IV A 2 Mers Seas Zeeen program SYSIASS.

†Université Lille Nord de France, LAGIS, UMR CNRS 8219, Cite Scientifique, BP 48, 59651 Villeneuve d'Ascq cedex, France. \{laurentiu.hetel\}@ec-lille.fr

$\ddagger$ Non-A, INRIA Lille-Nord Europe, France

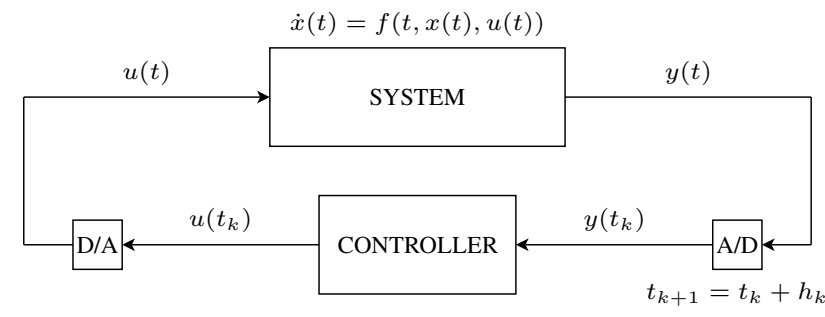

Fig. 1: Sampled-data system

\section{SAMPLED-DATA SYSTEMS}

The systems under consideration consist of a plant, a sampled-data control, and appropriate interface elements, such as represented in Figure 1, in which the blocks A/D and D/A correspond to an analog-to-digital converter (a sampler) and a digital-to-analog converter (a zero-order hold) respectively. There are various ways in which the controller synthesis for sampled-data systems can be done [7], [16]. One possible way is to design a continuous-time controller and then to approximate it using a sample-and-hold device. This approach is usually called emulation. Another approach is the discrete-time controller design where an exact or approximate discrete-time model of the plant is used to design a discrete-time controller, which will then be implemented to control the continuous-time plant using a zero-order-hold.

In this paper, the different concepts and results will mainly illustrated through the use of Linear Time-Invariant (LTI) sampled-data systems with linear state-feedback:

$$
\begin{aligned}
& \dot{x}(t)=A x(t)+B u(t), \forall t \geq 0, \\
& u(t)=K x\left(t_{k}\right), \quad \forall t \in\left[t_{k}, t_{k+1}\right), \quad k \in \mathbb{N},
\end{aligned}
$$

with $x$ the system state and $u$ the control signal, although some of the presented results have been originally given in more general control configurations or in the nonlinear case.

The following associated discrete-time model at instants $t_{k}$ is

$$
x_{k+1}=\Lambda\left(h_{k}\right) x_{k}, \forall k \in \mathbb{N}
$$

with $\Lambda(h):=\mathrm{e}^{A h}+\int_{0}^{h} \mathrm{e}^{A s} d s B K, x_{k}:=x\left(t_{k}\right)$. It is well known that for a constant sampling interval $h_{k}=T, \forall k \in$ $\mathbb{N}$, the discrete-time system (2) is asymptotically stable if and only if the matrix $\Lambda(T)$ is Schur, i.e. all its eigenvalues are strictly within the unit circle. However, in the case of time-varying sampling intervals, the analysis of sampled-data systems is quite complex, even in the LTI case. 
Example Consider the LTI sampled-data system (1) with

$$
A=\left[\begin{array}{cc}
0 & 1 \\
-2 & 0.1
\end{array}\right], B=\left[\begin{array}{l}
0 \\
1
\end{array}\right] K=\left[\begin{array}{ll}
1 & 0
\end{array}\right] .
$$

It is stable for both constant sampling intervals $T_{1}=1.5 \mathrm{~s}$ and $T_{2}=3 \mathrm{~s}$, as both matrices $\Lambda\left(T_{1}\right)$ and $\Lambda\left(T_{2}\right)$ are Schur. One may think that alternating the sampling interval between $T_{1}$ and $T_{2}$ will not affect the stability. However, the sampleddata system with periodically time-varying sampling intervals $T_{1} \rightarrow T_{2} \rightarrow T_{1} \rightarrow \cdots$ is unstable (see Figure 2, left). This is due to the fact that the Schurness of transition matrices is not preserved under matrix product, i.e. the matrix $\Lambda\left(T_{2}\right) \Lambda\left(T_{1}\right)$ is not Schur.

On the other hand, there may also exist stabilizing sampling sequences which are composed solely of sampling intervals corresponding to non-Schur matrices. For instance, the sampled-data system (1), (3) is unstable for both constant sampling periods $T_{3}=2.1 \mathrm{~s}$ and $T_{4}=4 \mathrm{~s}$, but it is stable under the periodically time-varying sampling $T_{3} \rightarrow T_{4} \rightarrow$ $T_{3} \rightarrow \cdots$ (see Figure 2, right). Indeed, the system transition matrix $\Lambda\left(T_{4}\right) \Lambda\left(T_{3}\right)$ is Schur.
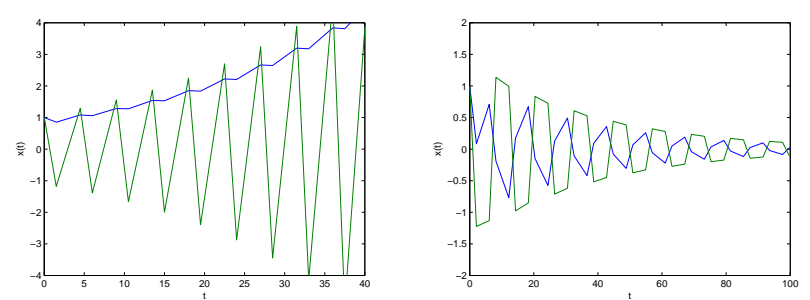

Fig. 2: Periodically time-varying sampling

Left: $T_{1}=1.5 s \rightarrow T_{2}=3 s \rightarrow T_{1} \rightarrow \cdots$ - Unstable

Right: $T_{3}=2.1 s \rightarrow T_{4}=4 s \rightarrow T_{3} \rightarrow \cdots$ - Stable

Thus, the following problems are raised:

- PROBLEM A: determine whether the sampled-data system is stable for any time-varying sampling interval $h_{k}$ with values in a bounded subset $\Omega \subseteq \mathbb{R}_{+}$.

- PROBLEM B: design a sampling law $h_{k}=$ $h\left(t, t_{k}, x\left(t_{k}\right), \cdots\right)$ that enlarges the sampling intervals while making the sampled-data system stable.

\section{QUALITATIVE PROPERTIES OF SAMPLED-DATA SYSTEMS}

The choice of sampling intervals is a critical issue in sampled-data implementations of emulated controllers. Intuitively, when the original continuous-time controller guarantees stability, by choosing a sufficiently fast sampling frequency, the stability will be preserved under sampleddata implementation. This conjecture has been confirmed in [35], for the case of input-affine nonlinear systems. The main result proves the fact that the discretization of stabilizing continuous-time nonlinear control laws with Lipschitz properties preserves the stability for constant and sufficiently small sampling intervals. This has been generalized in [10] to the case of time-varying sampling intervals, with dynamical control laws discretized using Euler approximation. In [78] it is shown that when the periodic sampling is sufficiently fast, the Input-to-State Stability (ISS) property of a nonlinear system is semi-globally practically preserved.

In [6], [8], [43], [59], some results concerning the emulation approach were generalized and unified in a methodological framework, by considering the preservation of dissipation inequality under sampling. It is shown that if a continuoustime controller provides some dissipation properties, then the resulting sampled-data system satisfies similar properties in a semi-global practical sense for sufficiently small sampling intervals.

\section{STABILITY ANALYSIS UNDER ARBITRARY TIME-VARYING SAMPLING}

This section concerns the estimation of the maximum allowable sampling intervals under arbitrary sampling variations.

\section{A. Time-delay approach}

This approach was initiated in [55] and further developed in [28] and in several other works [27], [41], [50], [75], [78], [85]. It consists in considering the discrete-time dynamics induced by the digital controller as a delay effect. For system (1), we may re-write $u(t)=K x\left(t_{k}\right)=K x(t-\tau(t))$, where the delay $\tau(t)=t-t_{k}, \forall t \in\left[t_{k}, t_{k+1}\right)$, is piecewise-linear, and satisfies $\dot{\tau}(t)=1$ for $t \neq t_{k}$, and $\tau\left(t_{k}\right)=0$. The LTI system with sampled data (1) is then re-modeled with a timevarying delay

$$
\dot{x}(t)=A x(t)+B K x(t-\tau(t)), \forall t \geq 0,
$$

In this context of delay systems [72], stability is studied using Lyapunov-Krasovskii or Lyapunov-Razumikhin functionals [32] depending on the past system state values. LMI stability criteria are given in [27], [28], [74]. For the nonlinear case, we point to the works in [41], [50], [78], [85].

\section{B. Hybrid system approach}

In this approach, the LTI sampled-data system (1) is modelled as an impulsive system (i.e. a dynamical system with discontinuous state variables, representing the sampling effect), with the state $\xi(t)=\left[x^{T}(t), z^{T}(t)\right]^{T}$, where $z(t)=$ $x\left(t_{k}\right), \forall t \in\left[t_{k}, t_{k+1}\right)$ :

$$
\left\{\begin{array}{l}
\dot{\xi}(t)=\left[\begin{array}{cc}
A & B K \\
0 & 0
\end{array}\right] \xi(t), \quad t \neq t_{k}, \forall k \in \mathbb{N}, \\
\xi\left(t_{k}\right)=\left[\begin{array}{l}
x\left(t_{k}^{-}\right) \\
x\left(t_{k}^{-}\right)
\end{array}\right], \quad t=t_{k}, \forall k \in \mathbb{N}
\end{array}\right.
$$

Stability analysis in this context involves Lyapunov functions with discontinuities at the impulse times [9], [58]. For nonlinear systems, the $\mathcal{L}_{p}$-stability properties have been studied in the more general context of networked control systems (NCS) [61]. See also [12], [62], [67], for a particularization to the sampled-data case. 


\section{Discrete-time approach and convex-embeddings}

For system (1) with time-varying sampling intervals in $[\underline{h}, \bar{h}], \underline{h}>0$, the convex-embedding approach [17], [29], [37], [31], uses the properties of the transition matrix $\Lambda(t-$ $\left.t_{k}\right)=e^{A\left(t-t_{k}\right)}+\int_{0}^{t-t_{k}} e^{A s} d s B K$, describing the evolution of $x(t)$ over the sampling interval with respect to the initial value $x\left(t_{k}\right)$. The idea is to express the stability problem as a finite number of LMIs, by embedding the matrix $\Lambda(\theta), \theta \in$ $[\underline{h}, \bar{h}]$ in a larger polytope $\overline{\mathcal{W}}:=\operatorname{conv}\left\{\Lambda_{i}, i=1, \cdots, N\right\}$ with finite number of vertices $\Lambda_{i}$. For quadratic Lyapunov functions $V(x)=x^{T} P x$, simple LMIs dependent on the polytope vertices may be obtained:

$$
P \succ 0, \Lambda_{i}^{T} P \Lambda_{i}-P \prec 0, \forall i \in\{1, \cdots, N\} .
$$

A continuous-time stability analysis of sampled-data systems based on convexification arguments has been proposed in [22], [38]. The discrete-time approach has also been considered in the case of nonlinear sampled-data systems [6], [42], [57], [60], [63]. However the developments are complex even in the case of periodic sampling.

\section{I/O approach}

In this approach, the sampling effect is seen as a perturbation $w(t)=x\left(t_{k}\right)-x(t)=-\int_{t_{k}}^{t} \dot{x}(\tau) d \tau$, and tools from robust control theory are used to guarantee the system's stability. The main idea is to write the sampled-data system (1) on each interval $\left[t_{k}, t_{k+1}\right)$ as the feedback interconnection of the operator $\Delta_{s h}: y \rightarrow w$ defined by: $w(t)=\left(\Delta_{s h} y\right)(t)=$ $-\int_{t_{k}}^{t} y(\tau) d \tau, \forall t \in\left[t_{k}, t_{k+1}\right)$, with an LTI system

$$
\mathbf{G}:=\left\{\begin{array}{l}
\dot{x}(t)=A_{c l} x(t)+B_{c l} w(t), \\
y(t)=\dot{x}(t),
\end{array}\right.
$$

where $A_{c l}=A+B K$ and $B_{c l}=B K$. The stability may be studied using classical robust control tools based on frequency domains analysis of the interconnection. See [40], [56], for a study based on the small gain theorem and [30] for a more general Integral Quadratic Constraints (IQCs) and Kalman-Yakubovich-Popov Lemma analysis. Extensions to nonlinear sampled-data systems have been provided in [64][66], [68] using dissipation theory.

\section{DYNAMIC CONTROL OF THE SAMPLING}

In this section, the sampling interval $h_{k}$ is considered as a control parameter. Increasing $h_{k}$ means reducing the quantity of information sent between the sensors and the actuators. In the literature, three main approaches cover the definition of such a sampling law $h_{k}=h\left(t, t_{k}, x\left(t_{k}\right), \cdots\right)$. In the first approach, the event-triggered control [13], [34], [48], [54], [77], [1], [79], [80], the system state is continuously monitored and the control actuation is performed only when certain events occur. These events are usually generated when the system state crosses a frontier in the state space. A dedicated hardware is required in order to monitor continuously the plant and generate such events. The second approach, the selftriggered control [45], [81], [82], [51], [52], [3], [2], [4], [5], aims at emulating event-triggered control without dedicated hardware, by pre-computing at each sampling instant the next admissible sampling interval based on previously received data and the knowledge of the plant dynamics. The third approach, the periodic event-triggered control (PET Control) [19], [70] considers uniform monitoring of the system state and event-triggering conditions that are verified periodically. In the following, we present a brief classification of some research directions in event- and self-triggered control.

\section{A. Deadband control approach [13], [69]}

The main idea of this event-triggered controller is that it is not necessary to update the control of the system when its state is close enough to the equilibrium point. The control is updated only when the state leaves some neighbourhood, called deadband. In [13] for example, the authors aim at reducing the number of actuations, while guaranteeing that the system state stays bounded. The chosen deadband is $\|C x\| \leq z_{\max }$ for some $z_{\max }>0$. In order to ensure that the disturbances will not make the output drift away from zero, the control outside the deadband is designed as $u(t)=-\operatorname{sgn}(C x(t))$. Inside the deadband, the control is $u(t)=\operatorname{sat}(K \hat{x}(t))$, and it is based on a simulation $\hat{x}(t)$ of the ideal evolution of the system, with initial conditions updated at triggering times $\left(\hat{x}\left(t_{k}\right)=x(t)\right.$ when $\left.\|C x\|=z_{\max }\right)$.

\section{B. Perturbation rejection approach [44], [48], [76]}

This type of event-triggered control scheme tries to take into account exogenous perturbations in event-triggering control by estimating and rejecting them. The sensors need to include an observer which estimates the perturbation. The event-generator used for this kind of controller is similar to the one used for deadband control (i.e. information is sent from the sensors to the actuators only when the state leaves a neighbourhood of the origin), except that, here, the error generating the trigger is computed with respect to the estimated state, instead of the equilibrium point. The events are thus generated when the measured state $x(t)$ leaves the vicinity $\Omega(\hat{x}(t))=\{x \mid\|x-\hat{x}(t)\| \leq \bar{e}\}$ of the estimated state $\hat{x}(t)$, for a given threshold $\bar{e}$.

\section{Lyapunov function levels approach [79]}

Another approach to event-triggered control consists in updating the control only when a chosen Lyapunov function crosses some predetermined energy levels. In [79], it is considered a nonlinear sampled-data system with an event generator defined by some levels of a Lyapunov function $V: V(x(t))=\eta V\left(x\left(t_{k}\right)\right)$, for some given scalar $0<\eta<$ 1 . Note that the trigger occurs when entering the region $V(x(t)) \leq \eta V\left(x\left(t_{k}\right)\right)$ therefore stability is not granted for all values of $\eta$. To ensure stability with such an event generator, it is necessary to guarantee that after each sampling instant $t_{k}$ there will be a time $t>t_{k}$ for which the eventtriggering condition $V(x(t))=\eta V\left(x\left(t_{k}\right)\right)$ will be satisfied. The method proposed in [79] consists in computing an upperbound $\eta^{*}$ of the minimal admissible $\eta$ (i.e. such that the previously mentionned property is satisfied for any $\eta \geq \eta^{*}>$ $0)$. A gridding of the state space is used to this aim. 


\section{Upper-bound on the system decay rate [52], [53]}

Unlike the Lyapunov function levels approach, here the sampling occurs when the state moves away from the equilibrium point, that is when the system does not satisfy a specified decay rate for a chosen Lyapunov function. Considering $V$, a Lyapunov function with exponential decayrate $\lambda_{0}$ for the closed-loop system with continuous feedback, and the map $\delta_{c}\left(x\left(t_{k}\right), t\right):=V(x(t))-V\left(x\left(t_{k}\right)\right) \mathrm{e}^{-\lambda\left(t-t_{k}\right)}$, for some $0<\lambda<\lambda_{0}$, the methods uses as a trigger the condition $\delta_{c}\left(x\left(t_{k}\right), t\right)=0$ for $t>t_{k}$. Note that implicitly, by construction, stability is granted in this approach. A selftriggering policy may be derived by computing online a Veronese embedding.

\section{E. ISS - approach [3], [4], [52], [77]}

ISS Lyapunov functions constitute another popular dynamic sampling control approach used to perform both eventtriggered and self-triggered control. It was initiated by [77], and further developed in [52], [3], [2] and [4]. The general approach in [77] considers the the reset system

$$
\left\{\begin{array}{l}
\dot{x}(t)=A x(t)+B K(x(t)-e(t)), \forall t \geq 0, \\
e\left(t_{k}\right)=0
\end{array}\right.
$$

where $e: \mathbb{R}_{+} \rightarrow \mathbb{R}^{n}$ is the measurement error between the current state and the last sampled state $(e(t)=x(t)-$ $\left.x\left(t_{k}\right)\right)$. The considered system is supposed to be ISS-stable with respect to the measurement error $e$, i.e. there exists a Lyapunov function $V$ such that $\frac{\partial V}{\partial x}(A x+B K(x+e)) \leq$ $-a\|x\|^{2}+b\|e\|\|x\|$ for some scalars $a$ and $b$. To enlarge the sampling intervals while ensuring stability, the control is updated when $b\|e(t)\|=a\|x(t)\|$. An extension to homogeneous systems, state-dependent homogeneous systems, and polynomial systems has been proposed in [3]. Further developments are proposed in [2] and [4], where the notion of isochronous manifolds is used to design the sampling function.

\section{F. $\mathcal{L}_{2}$-stability approach [81], [82]}

This approach is based on the notion of $\mathcal{L}_{2}$-stability and involves algebraic Riccati equations:

$$
0=P A+A^{T} P-P B B^{T} P+I+\frac{1}{\gamma^{2}} P E E^{T} P,
$$

where $\gamma$ represents the $\mathcal{L}_{2}$ gain. Considering the sampling error $e(t)=x(t)-x\left(t_{k}\right)$, and matrices $M$ and $N$ defined as: $M=\left(1-\beta^{2}\right) I+P B B^{T} P, N=\frac{1}{2}\left(1-\beta^{2}\right) I+P B B^{T} P$, it is shown that the $\mathcal{L}_{2}$-stability of the system is ensured with the event generator $e^{T}(t) M e(t)=x^{T}\left(t_{k}\right) N x\left(t_{k}\right)$. Furthermore, by analyzing the evolution of the term $e^{T}(t) M e(t)$ for $t \geq t_{k}$, it is possible to compute at each sampling instant a lower-bound estimation of the next allowable sampling interval, and thus perform a self-triggered control scheme. Differently from previous Lyapunov-based approaches, this approach allows the use of non-monotonously decreasing Lyapunov functions.

\section{G. Discrete-time and switched systems [18], [49]}

This approach considers the exact system discretization at sampling instants, given by (2). When the sampling interval $h_{k}$ is restricted to a finite number of values, the design of stabilizing sampling sequence $h_{k}$ can be related to the problem of designing a stabilizing switching law for a switched linear system [47]. This idea has been exploited in several publications using periodic [46] or state-dependent sampling laws [18], [49]. Alternatively, one may also find in the literature some approaches based on PET Control [19]. In this case, the discrete-time implementation of several triggering conditions (based on state error, $\mathcal{L}_{2}$ stability, decay of Lyapunov functions, etc.) leads to Piecewise Linear (PWL) discrete-time models. This allows the use of classical LMI-based approaches [20], [39] for analysing the system's stability and performance.

\section{H. Convex embeddings and state-dependent sampling [21],} [22], [24], [25]

This approach represents the counterpart of the convex embedding method [38] (used for analysing stability under arbitrary sampling) to the case of controlled sampling. It uses the properties of the transition matrix $\Lambda\left(t-t_{k}\right)=e^{A\left(t-t_{k}\right)}+$ $\int_{0}^{t-t_{k}} e^{A s} d s B K$ over the sampling interval $\left[t_{k}, t_{k+1}\right)$ in order to derive a sampling map defined by conic partitions of the state space. For the case of quadratic Lyapunov functions $V(x)=x^{T} P x$, the stability condition $\dot{V}(x(t)) \leq$ $-\beta V(x(t)), \forall t \in\left[t_{k}, t_{k}+h\left(x_{k}\right)\right)$ may be re-expressed in the form $x_{k}^{T} \Phi(\theta) x_{k} \leq 0$, for all $\theta \in\left[0, h\left(x_{k}\right)\right)$ where $x_{k}=x\left(t_{k}\right)$ and

$$
\Phi(\theta)=\left[\begin{array}{c}
\Lambda(\theta) \\
I
\end{array}\right]^{T}\left[\begin{array}{cc}
A^{T} P+P A+\beta P & P B K \\
* & 0
\end{array}\right]\left[\begin{array}{c}
\Lambda(\theta) \\
I
\end{array}\right] .
$$

Stabilizing sampling maps may be designed using the fact that a sampling interval $h\left(x_{k}\right)=\tau$ ensures the decay of the Lyapunov function $V(x)$ in the region

$$
\mathcal{Z}(\tau)=\left\{x \in \mathbb{R}^{n}: x^{T} \Phi(\theta) x \leq 0, \forall \theta \in[0, \tau]\right\} .
$$

For practical implementations, considering a polytopic approximation with $N$ vertices $\Phi_{i}(\tau), i=1, \cdots, N$, such that $\Phi(\theta) \in \operatorname{conv}\left\{\Phi_{i}(\tau), i=1, \cdots, N\right\}$ for all $\theta \in[0, \tau], \mathcal{Z}(\tau)$ may be over-approximated by a finite intersection of conic regions

$$
\hat{\mathcal{Z}}(\tau)=\left\{x \in \mathbb{R}^{n}: x^{T} \Phi_{i} x \leq 0, \forall i=1, \ldots, N\right\} \supseteq \mathcal{Z}(\tau) .
$$

A stabilizing sampling map $h(x)$ may be defined as

$$
h\left(x_{k}\right)=\max \left\{\tau \in \mathbb{R}^{+}: x_{k} \in \hat{\mathcal{Z}}(\tau)\right\} .
$$

The approach allows to provide some optimisation in the design of sampling maps by optimising the choice of Lyapunov functions using LMI based tools. It is possible, for example, to optimise the choice the the Lyapunov function $V(x)=x^{T} P x$ which enlarges the lower bound of the 
sampling map $h\left(x_{k}\right)$, by solving the optimization problem

$$
\begin{gathered}
\sup \tau \text { such that } \\
\exists P \succ 0 \\
\qquad(\theta)=\left[\begin{array}{c}
\Lambda(\theta) \\
I
\end{array}\right]^{T}\left[\begin{array}{cc}
A^{T} P+P A+\beta P & P B K \\
* & 0
\end{array}\right]\left[\begin{array}{c}
\Lambda(\theta) \\
I
\end{array}\right] \prec 0 \\
\forall \theta \in[0, \tau]
\end{gathered}
$$

which may also be transformed in a finite set of LMIs using polytopic approximations of $\Phi(\tau)$.

This optimization procedure may also be used to analyse the system's stability with given conic regions $\mathcal{R}_{i}$ of the state-space and associated bounds on the sampling intervals $\tau_{i}$. This problem setting is called state-dependent sampling. In this context, convex embeddings have been used in [22], [24], and Lyapunov-Krasovskii functionals in [23].

\section{CONCLUSION}

This tutorial has presented some of the basic concepts and recent research directions in sampled-data systems: time-delay, hybrid, discrete-time and input-output models for sampled-data systems; stability for systems with arbitrary sampling intervals; design of stabilizing state-dependent sampling laws. It is to be emphasized that this brief overview is far from being an exhaustive survey on the stability of sampled-data systems with time-varying sampling intervals. Such a research topic is still wide open and continuously growing. Unavoidably, not all possible results are mentioned here.

\section{REFERENCES}

[1] A. Albert. Comparison of event-triggered and time-triggered concepts with regard to distributed control systems. Embedded World, 55(9):235-252, 2004.

[2] A. Anta and P. Tabuada. Isochronous manifolds in self-triggered control. In 48th IEEE Conference on Decision and Control, pages 3194-3199, Shanghai, China, 2009.

[3] A. Anta and P. Tabuada. To sample or not to sample: self-triggered control for nonlinear systems. IEEE Transactions on Automatic Control, 55(9):2030-2042, 2010.

[4] A. Anta and P. Tabuada. Exploiting isochrony in self-triggered control. IEEE Transactions on Automatic Control, 57(4):950-962, 2012.

[5] J. Araujo, A. Anta, M. Mazo Jr., J. Faria, A. Hernandez, P. Tabuada, and K.-H. Johansson. Self-triggered control over wireless sensor and actuator networks. In International Conference on Distributed Computing in Sensor Systems, Barcelona, Spain, 2011.

[6] A. Astolfi, D. Nesic, and A.R. Teel. Trends in nonlinear control. In 47th IEEE Conference on Decision and Control, pages 1870 - 1882, 2008.

[7] K.-J. Aström and B. Wittenmark. Computer controlled systems: theory and design. Prentice Hall, 1996.

[8] H. Beikzadeh and H.J. Marquez. Dissipativity of nonlinear multirate sampled-data systems under emulation design. Automatica, 49(1):308 $-312,2013$.

[9] C. Briat and A. Seuret. A looped-functional approach for robust stability analysis of linear impulsive systems. Systems \& Control Letters, 61(10):980 - 988, 2012.

[10] L. Burlion, T. Ahmed-Ali, and F. Lamnabhi-Lagarrigue. On the stability of a class of nonlinear hybrid systems. Nonlinear Analysis: Theory, Methods \& Applications, 65(12):2236 - 2247, 2006.

[11] G. Buttazzo and L. Abeni. Adaptive workload management through elastic scheduling. Real-Time Systems, 23(1):7-24, 2002.

[12] D. Carnevale, A.R. Teel, and D. Nešić. A Lyapunov proof of an improved maximum allowable transfer interval for networked control systems. IEEE Transactions on Automatic Control, 52(5):892 - 897, 2007.
[13] A. Cervin and K.-J. Aström. On limit cycles in event-based control systems. In 46th IEEE Conference on Decision and Control, pages 3190-3195, New Orleans, Louisiana, USA, 2007.

[14] A. Cervin, J. Eker, B. Bernhardsson, and K.-E. Arzen. Feedbackfeedforward scheduling of control tasks. Real-Time Systems, 23(1):2553, 2002.

[15] J. Chen, K.-H. Johansson, S. Olariu, I.-C. Paschalidis, and I. Stojmenovic. Guest editorial special issue on wireless sensor and actuator networks. IEEE Transactions on Automatic Control, 56(10):2244 2246, 2011.

[16] T. Chen and B. Francis. Optimal sampled-data control systems. Springer, 1993.

[17] M.B.G. Cloosterman, L. Hetel, N. van de Wouw, W.P.M.H. Heemels, J. Daafouz, and H. Nijmeijer. Controller synthesis for networked control systems. Automatica, 46(10):1584-1594, 2010.

[18] G.-S Deaecto, M. Souza, and J.-C. Geromel. State feedback switched control of discrete-time switched linear systems with application to networked control. In 21st Mediterranean Conference on Control \& Automation, pages 877-883, 2013.

[19] M.C.F. Donkers and W.P.M.H. Heemels. Output-based event-triggered control with guaranteed -gain and improved event-triggering. In 49th IEEE Conference on Decision and Control, pages 3246-3251, 2010.

[20] G. Ferrari-Trecate, F.-A. Cuzzola, D. Mignone, and M. Morari. Analysis of discrete-time piecewise affine and hybrid systems. Automatica, 38(12):2139-2146, 2002.

[21] C. Fiter, L. Hetel, W. Perruquetti, and J.-P. Richard. State dependent sampling: an LMI based mapping approach. In 18th IFAC World Congress, pages 8824-8829, Milan, Italy, 2011.

[22] C. Fiter, L. Hetel, W. Perruquetti, and J.-P. Richard. A state dependent sampling for linear state feedback. Automatica, 48(8):1860-1867, 2012.

[23] C. Fiter, L. Hetel, W. Perruquetti, and J.-P. Richard. State-dependent sampling for perturbed time-delay systems. In 51st IEEE Conference on Decision and Control, pages 2358-2363, Maui, Hawaii, USA, 2012.

[24] C. Fiter, L. Hetel, W. Perruquetti, and J.-P. Richard. A robust polytopic approach for state-dependent sampling. In 12th European Control Conference, pages 2603-2608, Zurich, Switzerland, 2013.

[25] C. Fiter, L. Hetel, W. Perruquetti, and J.-P. Richard. A self-triggered control based on convex embeddings for perturbed LTI systems. In 9th IFAC Symposium on Nonlinear Systems, Toulouse, France, 2013.

[26] G. Franklin, D. Powell, and M. Workman. Digital Control of Dynamic Systems. Addison-Wesley Longman Publishing Co., Inc., 3rd edition, 1997.

[27] E. Fridman. A refined input delay approach to sampled-data control. Automatica, 46(2):421-427, 2010.

[28] E. Fridman, A. Seuret, and J.-P Richard. Robust sampled-data stabilization of linear systems: An input delay approach. Automatica, 40(8):1441-1446, 2004.

[29] H. Fujioka. A discrete-time approach to stability analysis of systems with aperiodic sample-and-hold devices. IEEE Transactions on Automatic Control, 54(10):2440-2445, 2009.

[30] H. Fujioka. Stability analysis of systems with aperiodic sample-andhold devices. Automatica, 45(3):771-775, 2009.

[31] R. Gielen, S. Olaru, M. Lazar, W. Heemels, N. Van de Wouw, and S.-I. Niculescu. On polytopic inclusions as a modeling framework for systems with time-varying delays. Automatica, 46(3):615-619, 2010.

[32] K. Gu, V. Kharitonov, and J. Chen. Stability of time-delay systems. Boston: Birkhauser, 2003

[33] W.P.M.H. Heemels, K.-H Johansson, and P. Tabuada. An introduction to event-triggered and self-triggered control. In 51st IEEE Conference on Decision and Control, pages 3270-3285, 2012.

[34] W.P.M.H Heemels, J.-H. Sandee, and P.P.J. Van Bosch. Analysis of event-driven controllers for linear systems. International Journal of Control, 81(4):571-590, 2008.

[35] G. Herrmann, S.K. Spurgeon, and C. Edwards. Discretization of sliding mode based control schemes. In Proceedings of the 38th IEEE Conference on Decision and Control, volume 5, pages 4257 - 4262, 1999.

[36] J.-P. Hespanha, P. Naghshtabrizi, and Y. Xu. A survey of recent results in networked control systems. IEEE Special Issue on Technology of Networked Control Systems, 95(1):138-162, 2007.

[37] L. Hetel, J. Daafouz, and C. Iung. Stabilization of arbitrary switched linear systems with unknown time-varying delays. IEEE Transactions on Automatic Control, 51(10):1668-1674, 2006. 
[38] L. Hetel, A. Kruszewski, W. Perruquetti, and J.-P Richard. Discrete and intersample analysis of systems with aperiodic sampling. IEEE Transactions on Automatic Control, 56(7):1696-1701, 2011.

[39] M. Johansson. Piecewise linear control systems. Springer, 2003.

[40] C.-Y. Kao and B. Lincoln. Simple stability criteria for systems with time-varying delays. Automatica, 40(8):1429 - 1434, 2004.

[41] I. Karafyllis and M. Krstic. Nonlinear stabilization under sampled and delayed measurements, and with inputs subject to delay and zero-order hold. IEEE Transactions on Automatic Control,, 57(5):1141 - 1154, 2012.

[42] D. Laila, D. Nešić, and A. Astolfi. 3 sampled-data control of nonlinear systems. In Antonio Loría, Françoise Lamnabhi-Lagarrigue, and Elena Panteley, editors, Advanced Topics in Control Systems Theory, volume 328 of Lecture Notes in Control and Information Science, pages 91137. Springer London, 2006.

[43] D. Laila, D. Nešić, and A.R. Teel. Open- and closed-loop dissipation inequalities under sampling and controller emulation. European Journal of Control, 8(2):109 - 125, 2002.

[44] D. Lehmann and J. Lunze. Event-based control with communication delays. In 18th IFAC World Congress, Milan, Italy, 2011.

[45] M.-D. Lemmon, T. Chantem, X.-S. Hu, and M. Zyskowski. On selftriggered full-information h-infinity controllers. In 10th International Conference on Hybrid Systems: Computation and Control, Pisa, Italy, 2007.

[46] X.-G. Li, A. Cela, S. Niculescu, and A. Reama. Some problems in the stability of networked-control systems with periodic scheduling. International Journal of Control, 83(5):996-1008, 2010.

[47] D. Liberzon. Switching in systems and control. Springer, 2003.

[48] J. Lunze and D. Lehmann. A state-feedback approach to event-based control. Automatica, 46(1):211-215, 2010

[49] S. Maalej, C. Fiter, L. Hetel, and J.-P. Richard. State-dependent sampling for linear time invariant systems: a discrete time analysis. In 20th IEEE Mediterranean Conference on Control and Automation, pages 1129-1134, Barcelona, Spain, 2012.

[50] F. Mazenc, M. Malisoff, and T.N. Dinh. Robustness of nonlinear systems with respect to delay and sampling of the controls. Automatica, 49(6): 1925 - 1931, 2013.

[51] M. Mazo Jr., A. Anta, and P. Tabuada. On self-triggered control for linear systems: guarantees and complexity. In European Control Conference, Budapest, Hungary, 2009.

[52] M. Mazo Jr., A. Anta, and P. Tabuada. An ISS self-triggered implementation of linear controllers. Automatica, 46(8):1310-1314, 2010.

[53] M. Mazo Jr. and P. Tabuada. Input-to-state stability of self-triggered control systems. In 48th IEEE Conference on Decision and Control, pages 928-933, Shanghai, China, 2009.

[54] M. Mazo Jr. and P. Tabuada. Decentralized event-triggered control over wireless sensor/actuator networks. IEEE Transactions on Automatic Control, 56(10):2456-2461, 2011.

[55] Y. Mikheev, V. Sobolev, and E. Fridman. Asymptotic analysis of digital control systems. Automation and Remote Control, 49(9):1175-1180, 1988.

[56] L. Mirkin. Some remarks on the use of time-varying delay to model sample-and-hold circuits. IEEE Transactions on Automatic Control, 52(6):1109-1112, 2007.

[57] S. Monaco, D. Normand-Cyrot, and C. Califano. From chronological calculus to exponential representations of continuous and discrete-time dynamics: A Lie-algebraic approach. IEEE Transactions on Automatic Control, 52(12):2227 - 2241, 2007.

[58] P. Naghshtabrizi, J.-P. Hespanha, and A.-R. Teel. Exponential stability of impulsive systems with application to uncertain sampled-data systems. Systems and Control Letters, 57(5):378-385, 2008.

[59] D. Nešić and A.R. Teel. Sampled-data control of nonlinear systems: An overview of recent results. In S.O.Reza Moheimani, editor, Perspectives in robust control, volume 268 of Lecture Notes in Control and Information Sciences, pages 221-239. Springer London, 2001.

[60] D. Nešić and A.R. Teel. A framework for stabilization of nonlinear sampled-data systems based on their approximate discrete-time models. IEEE Transactions on Automatic Control, 49(7):1103 - 1122, 2004.

[61] D. Nešić and A.R. Teel. Input-output stability properties of networked control systems. IEEE Transactions on Automatic Control, 49(10): 1650 - 1667, 2004.

[62] D. Nešić, A.R. Teel, and D. Carnevale. Explicit computation of the sampling period in emulation of controllers for nonlinear sampled-data systems. IEEE Transactions on Automatic Control, 54(3):619 - 624, 2009.

63] D. Nešić, A.R. Teel, and P.V. Kokotović. Sufficient conditions for stabilization of sampled-data nonlinear systems via discrete-time approximations. Systems \& Control Letters, 38(45):259 - 270, 1999.

64] H. Omran, L. Hetel, and J.-P. Richard. Local stability of bilinear systems with asynchronous sampling. In 4th IFAC Conference on Analysis and Design, Eindhoven, The Netherlands, 2012.

[65] H. Omran, L. Hetel, J. P. Richard, and F. Lamnabhi-Lagarrigue. Stabilité des systèmes non linéaires sous échantillonnage apériodique. Journal Européen des Systèmes Automatisés. To appear

[66] H. Omran, L. Hetel, J.-P. Richard, and F. Lamnabhi-Lagarrigue. Stability analysis of bilinear systems under aperiodic sampled-data control. Automatica. To appear.

[67] H. Omran, L. Hetel, J.-P. Richard, and F. Lamnabhi-Lagarrigue. Stability of bilinear sampled-data systems with an emulation of static state feedback. In IEEE 51st Annual Conference on Decision and Control, pages 7541 - 7546, 2012.

[68] H. Omran, L. Hetel, J.-P. Richard, and F. Lamnabhi-Lagarrigue. On the stability of input-affine nonlinear systems with sampled-data control. In European Control Conference (ECC), pages 2585 - 2590, 2013.

[69] P.-G. Otanez, J.-R. Moyne, and D.-M. Tilbury. Using deadbands to reduce communication in networked control systems. In American Control Conference, pages 3015-3020, Anchorage, Alaska, USA, 2002.

[70] R. Postoyan, A. Anta, W.P.M.H. Heemels, P. Tabuada, and D. Nesic. Periodic event-triggered control for nonlinear systems. In 52nd IEEE Conference on Decision and Control, CDC 2013, 2013.

[71] K. Åström and B. Wittenmark. Computer-controlled systems: theory and design. Prentice-Hall information and system sciences series. Prentice-Hall, Upper Saddle River, NJ, 1997.

[72] J.-P Richard. Time delay systems: an overview of some recent advances and open problems. Automatica, 39(10):1667-1694, 2003.

[73] J.-P. Richard and T. Divoux. Systèmes commandés en réseau. IC2 Systèmes Automatisés. Hermès-Lavoisier, 2007.

[74] A. Seuret. A novel stability analysis of linear systems under asynchronous samplings. Automatica, 48(1):177-182, 2012.

[75] A. Seuret and J.P. Richard. Control of a remote system over network including delays and packet dropout. In 17th IFAC World Congress, 2008.

[76] C. Stöcker and J. Lunze. Event-based control of input-output linearizable systems. In 18th IFAC World Congress, Milan, Italy, 2011.

[77] P. Tabuada. Event-triggered real-time scheduling of stabilizing control tasks. IEEE Transactions on Automatic Control, 52(9):1680-1685, 2007.

[78] A.R. Teel, D. Nesic, and P.V. Kokotović. A note on input-to-state stability of sampled-data nonlinear systems. In Proceedings of the 37th IEEE Conference on Decision and Control, volume 3, pages 2473 2478, 1998 .

[79] M. Velasco, P. Marti, and E. Bini. On lyapunov sampling for eventdriven controllers. In 48th IEEE Conference on Decision and Control, pages 6238-6243, Shanghai, China, 2009.

[80] X. Wang and M.-D. Lemmon. Event design in event-triggered feedback control systems. In 47th IEEE Conference on Decision and Control, pages 2105-2110, Cancun, Mexico, 2008.

[81] X. Wang and M.-D. Lemmon. Self-triggered feedback control systems with finite-gain $\mathcal{L}_{2}$ stability. IEEE Transactions on Automatic Control, 54(3):452-467, 2009.

[82] X. Wang and M.-D. Lemmon. Self-triggering under state-independent disturbances. IEEE Transactions on Automatic Control, 55(6):14941500, 2010.

[83] B. Wittenmark, J. Nilsson, and M. Torngren. Timing problems in real-time control systems. In American Control Conference, pages 2000-2004, Seattle, Washington, USA, 1995.

[84] W. Zhang, M.S. Branicky, and S.M. Phillips. Stability of networked control systems. IEEE Control Systems Magazine, 21(1):84-99, 2001.

[85] Y. Zheng, D. Owens, and S. Billings. Fast sampling and stability of nonlinear sampled-data systems: Part 2. The IMA Journal of Mathematical Control \& Information, 7:13 - 33, 1990. 